\title{
12 Klimawandel und Gesundheit: Welche Rolle spielt der Klimawandel im Gesundheits- bewusstsein der Befragten? Ergebnisse einer deutschlandweiten Bevölkerungsbefragung
}

\author{
Caroline Schmuker, Bernt-Peter Robra, Kai Kolpatzik, Klaus Zok und \\ Jürgen Klauber
}

C. Günster | I. Klauber | B.P. Robra | C. Schmuker | A. Schneider (Hrsg.) Versorgungs-Report Klima und Gesundheit. DOI 10.32745/9783954666270-12, ㅇ MWV Medizinisch Wissenschaftliche Verlagsgesellschaft Berlin 2021

Der vorliegende Beitrag berichtet über die Ergebnisse einer bundesweiten Onlinebefragung $(n=3.006)$ des Wissenschaftlichen Institutes der AOK (WIdO) zu zentralen Fragestellungen des Klima- und Gesundheitsbewusstseins in der Bevölkerung. Im Fokus der Befragung standen Umweltbelastungen, die im Zuge des Klimawandels an Bedeutung gewinnen werden: Hitze, UV-Strahlung, Luftschadstoffe und Pollenallergene. Teilnehmende wurden nach ihrem Informationsstand, nach gesundheitlichen Beeinträchtigungen, zu ihrem Schutzverhalten und zur Nutzung von Warn- und Informationsdiensten befragt. Die Ergebnisse zeigen, dass weiterhin deutlicher Informationsbedarf zu den gesundheitlichen Risiken des Klimawandels in der Gesellschaft besteht. Das Schutzverhalten in der Bevölkerung ist verbesserungsfähig, gerade auch mit Blick auf ältere oder gesundheitlich belastete Personen, die besonders gefährdet sind. Es besteht eine erhebliche kommunikative Herausforderung auf dem Weg, das individuelle präventive Verhalten zu stärken, insbesondere bei weniger sichtbaren Umweltbelastungen wie erhöhten UV-Strahlungen oder Luftschadstoffen, die von vielen Befragten auch nicht als beeinträchtigend erlebt werden. Die Bekanntheit und Nutzung von vorhandenen Informations- und Frühwarnsystemen ist noch sehr gering ausgeprägt.
This publication reports the results of a nationwide online survey $(n=3.006)$ conducted by the Scientific Institute of the General Health Insurance funds (WIdO) addressing the awareness of health aspects of climate change. The survey focuses on climate related health risks that are expected to gain significance in the progress of climate change: heat, UV-radiation, airborne pollutants and pollen allergens. Participants were asked about their level of information on health risks, any health impairment experienced, their adaption measures, and the use of warning and information systems. The results clearly show further need of information about climate related health risks. Adaption behaviour should be improved, especially with regard to vulnerable persons, e.g. those with impaired health or of older age. Communicating climate related health risks and adaption behaviour to the public is especially challenging since risks such as UV or some airborne pollutants do not always cause immediate subjective impairments to humans. The awareness and use of existing information and warning systems is still very low. 
II Gesundheitliche Auswirkungen des Klimawandels und Herausforderungen

für die medizinische Versorgung in Deutschland

\subsection{Einleitung}

Der vorliegende Beitrag stellt die Ergebnisse einer bundesweiten Bevölkerungsbefragung zum Thema Klimawandel und Gesundheit dar, die das Wissenschaftliche Institut der AOK (WIdO) im September des Jahres 2020 online durchgeführt hat. Verschiedene internationale und nationale Forschungsinstitutionen haben in den letzten Jahrzehnten die Auswirkungen des Klimawandels auf die Lebensbedingungen und auf die Gesundheit der Menschen erforscht. Seitdem ist die Verbindung zwischen Klimawandel und Gesundheit auch in Deutschland zunehmend Gegenstand von Forschung, Medienberichterstattung und gesundheitspolitischem Handeln.

Ziel der vorliegenden Befragung war eine Bestandsaufnahme zu zentralen Fragestellungen des Gesundheits- und Klimabewusstseins in der Bevölkerung:

- Informationsstand und Risikoeinschätzung: Wie gut sind die Befragten über mit dem Klimawandel verbundene veränderte Umweltbedingungen und deren mögliche Auswirkungen auf die Gesundheit informiert? Wie besorgt sind die Befragten dabei über ihre eigene Gesundheit?

- subjektive Beeinträchtigungen durch Umwelteinflüsse: In welchem Ausmaß erleben die Befragten klimaassoziierte Umwelteinflüsse (Hitze, UV-Strahlung, Luftschadstoffe und Allergene [Pollen]) als belastend? Welche Bevölkerungsgruppen mit erhöhter Belastung können identifiziert werden?

- Klimaanpassungsverhalten: Wie verbreitet sind Verhaltensweisen zum Schutz vor bzw. zur Anpassung an erhöhte Belastungen im Kontext von Klimaänderungen?

- Stellenwert der Warn- und Informationsdienste: In welchem Umfang erreichen Informationen und Vorhersagen zu Umweltbelastungen die Gesamtbevölkerung?

Im Fokus der Befragung stehen vier klimaassoziierte Umwelteinflüsse, die von hoher gesund- heitlicher Relevanz sind und nach aktuellen Erkenntnissen im Zuge des Klimawandels an Bedeutung gewinnen werden. Das sind: eine $\mathrm{Zu}-$ nahme an Hitzewellen sowie eine erhöhte Exposition gegenüber Luftschadstoffen (z.B. Feinstaub, Ozon), UV-Strahlung und Pollenallergenen (ausführlicher hierzu s. Kap. 3, 8, 9 sowie 10).

\subsection{Methoden}

\subsubsection{Datenerhebung und Stichprobe}

Die vorliegenden Ergebnisse basieren auf einer repräsentativen Online-Befragung der deutschsprachigen Wohnbevölkerung im Alter von 18 bis 86 Jahren mit Internet-Zugang. Es handelt sich um eine regional repräsentative Quotenstichprobe von insgesamt 3.006 Personen. Die Fallzahlen je Bundesland wurden auf Basis der Einwohnerzahlen nach Mikrozensus berechnet. Innerhalb jedes Bundeslandes greift dann eine vorgegebene Quotierung nach Alter und Geschlecht, sodass die Stichprobe in jedem Bundesland als alters- und geschlechtsrepräsentativ anzusehen ist.

Die Befragung wurde durch die Respondi AG (Köln) im Zeitraum vom o1. bis zum 17. September 2020 durchgeführt. Respondi verfügt über ein eigenes Online-Access-Panel mit über 100.0oo aktiven Teilnehmenden deutschlandweit, das primär für Marktforschung genutzt wird. Das Panel wird von einem professionellen Panelteam verwaltet und über Scoring- und Kontrollverfahren einem permanenten Qualitätscontrolling unterzogen. Teilnehmende werden per Zufallsverfahren für Befragungen ausgewählt, eine Selbstselektion ist nicht möglich. Der Anteil der realisierten Teilnahmen (3.006) an allen eingeladenen Personen (12.967) lag bei 23,2\% . 214 (1,7\%) der Eingeladenen haben den Fragebogen abgebrochen, 1.699 (13,1\%) Teilnahmen wurden beendet, weil die Quotenkriterien bereits erfüllt waren und 8 eingeladene Personen $(0,1 \%)$ erfüllten die Selektionskrite- 
12 Klimawandel und Gesundheit: Welche Rolle spielt der Klimawandel im Gesundheitsbewusstsein der Befragten? Ergebnisse einer deutschlandweiten Bevölkerungsbefragung

rien zur Teilnahme nicht. In die Auswertung gehen nur vollständig abgeschlossene Fragebögen ein. Unvollständige Fragebögen werden als abgebrochen gewertet.

\subsubsection{Entwicklung des Fragebogens}

Das Konzept der Befragung wurde im Wissenschaftlichen Institut der AOK (WIdO) mit Unterstützung der Autoren entwickelt. Hierfür wurden validierte Fragen aus themenverwandten Umfragen zusammengestellt, sowie eigene Fragen entlang der Leitfragen ergänzt. Inhaltlich ist der Fragebogen wie folgt aufgebaut:

Teil 1: Der erste Teil des Fragebogens enthält allgemeine Fragen zum Gesundheitszustand und zu Risikofaktoren, die im Zusammenhang mit Umwelteinflüssen bekannt sind. Neben Alter, Geschlecht und ausgewählten chronischen Erkrankungen wird auch der subjektive (selbst eingeschätzte) Gesundheitszustand erfasst. Hierfür wurden die Frageformulierungen aus der Studie „Gesundheit in Deutschland aktuell“ (GEDA 2014/2015-EHIS, Robert Koch-Institut 2014) verwendet.

Teil 2: Der zweite Teil des Fragebogens besteht aus vier Fragemodulen zu klimaassoziierten Umweltfaktoren: Hitze, UV-Strahlung, Pollen und Luftschadstoffe. Die Module enthalten jeweils Fragen $\mathrm{zu}$ wahrgenommenen Beeinträchtigungen, zu körperlichen Beschwerden, zum Schutz- und Anpassungsverhalten sowie zur Nutzung von Warn- und Informationsdiensten. Ein Teil der Fragen (insbesondere zu Hitzebelastung und Schutzverhalten) orientiert sich an der Befragung „Klimawandel in Leipzig“ (Stadt Leipzig 2018), einer städtischen Bürgerbefragung zu gesundheitlichen und stadträumlichen Aspekten des Klimawandels. Die Fragen zu Warn- und Informationssystemen orientieren sich an einer Befragung des Umweltbundesamtes (UBA) im Rahmen der „Evaluation von Informationssystemen zu Klimawandel und Gesundheit" (Capellaro u. Sturm 2015).
Teil 3: Im dritten Teil des Fragebogens werden übergreifende Aspekte zum Thema Klimawandel und Gesundheit erhoben, darunter der allgemeine Informationsstand, Sorgen und Ängste, sowie Einstellungen zum Klimaschutz und zum klimafreundlichen Verhalten.

Teil 4: Der Fragebogen schließt mit der Erhebung der soziodemografischen Merkmale.

Um die Fragebogenführung zu vereinfachen, wurden abschließend nach eigenem Ermessen Frageformulierungen angepasst und Antwortkategorien vereinheitlicht. Zur Qualitätssicherung wurde der Gesamtfragebogen mehrfach in Hinblick auf Verständlichkeit, Filterführung, Länge und Schlüssigkeit getestet und den Rückmeldungen entsprechend angepasst. Zudem wurden die ersten 100 teilnehmenden Personen nach Start der Feldphase gebeten, sich am Ende des Fragebogens zur Handhabung des Fragebogens zu äußern. Das Feedback der Teilnehmer war durchgängig positiv, sodass keine weiteren Anpassungen notwendig waren.

\subsubsection{Statistische Auswertungen}

Die Antworten wurden größtenteils in Form verbalisierter Likert-Skalen erfasst. Für die bessere Lesbarkeit werden Anteilswerte im Folgenden gerundet ausgewiesen. Die statistische Signifikanz von Gruppenunterschieden wurde mit dem Chi-Quadrat-Test nach Pearson überprüft. Es wird von einem statistisch signifikanten Unterschied zwischen Gruppen ausgegangen, wenn der entsprechende $\mathrm{p}$-Wert kleiner als 0,05 ist.

\subsubsection{Repräsentativität der Stichprobe}

Tabelle 1 zeigt die Zusammensetzung der Stichprobe $(\mathrm{n}=3.006)$ nach soziodemografischen und gesundheitsbezogenen Merkmalen. Die Stichprobe ist aufgrund der festgelegten Quoten alters- und geschlechtsrepräsentativ für die 
II Gesundheitliche Auswirkungen des Klimawandels und Herausforderungen für die medizinische Versorgung in Deutschland

Tab. 1 Beschreibung der Stichprobe anhand soziodemografischer und gesundheitsbezogener Merkmale

\begin{tabular}{|c|c|c|}
\hline & Häufigkeit (N) & Prozente (\%) \\
\hline Gesamtzahl der Befragten & 3.006 & $100,0 \%$ \\
\hline \multicolumn{3}{|l|}{ Alter in Jahren } \\
\hline 18 bis 39 & 982 & $32,7 \%$ \\
\hline 40 bis 59 & 1.078 & $35,9 \%$ \\
\hline 60 und älter & 946 & $31,5 \%$ \\
\hline \multicolumn{3}{|l|}{ Geschlecht } \\
\hline Frauen & 1.513 & $50,3 \%$ \\
\hline Männer & 1.493 & $49,7 \%$ \\
\hline \multicolumn{3}{|l|}{ Bildungsstand* } \\
\hline niedrig & 309 & $10,3 \%$ \\
\hline mittel & 1.649 & $54,9 \%$ \\
\hline hoch & 1.048 & $34,9 \%$ \\
\hline \multicolumn{3}{|l|}{ Region } \\
\hline städtisch & 1.513 & $50,3 \%$ \\
\hline mit Verstädterungsansätzen & 497 & $16,5 \%$ \\
\hline ländlich & 996 & $33,1 \%$ \\
\hline \multicolumn{3}{|l|}{ chronische Erkrankungen } \\
\hline ja & 1.519 & $50,5 \%$ \\
\hline nein & 1.487 & $49,5 \%$ \\
\hline \multicolumn{3}{|l|}{ subjektiver Gesundheitszustand } \\
\hline sehr gut/gut & 1.954 & $65,0 \%$ \\
\hline mittelmäßig & 802 & $26,7 \%$ \\
\hline schlecht/sehr schlecht & 250 & $8,3 \%$ \\
\hline \multicolumn{3}{|l|}{ ärztlich diagnostizierte Erkrankungen } \\
\hline Atemwegserkrankungen & 537 & $17,9 \%$ \\
\hline - davon Asthma & 349 & $11,6 \%$ \\
\hline - davon chronische Lungenerkrankungen & 295 & $9,8 \%$ \\
\hline Pollenallergie & 679 & $22,6 \%$ \\
\hline Herz-Kreislauf-Erkrankungen & 330 & $11,0 \%$ \\
\hline Hauterkrankungen & 361 & $12,0 \%$ \\
\hline - davon Neurodermitis & 245 & $8,2 \%$ \\
\hline - davon Hautkrebs & 145 & $4,8 \%$ \\
\hline \multicolumn{3}{|l|}{ Staatsangehörigkeit } \\
\hline deutsch & 2.924 & $97,3 \%$ \\
\hline andere & 82 & $2,7 \%$ \\
\hline
\end{tabular}

*operationalisiert über die Variablen Schulbildung und berufliche Ausbildung 
12 Klimawandel und Gesundheit: Welche Rolle spielt der Klimawandel im Gesundheitsbewusstsein der Befragten? Ergebnisse einer deutschlandweiten Bevölkerungsbefragung

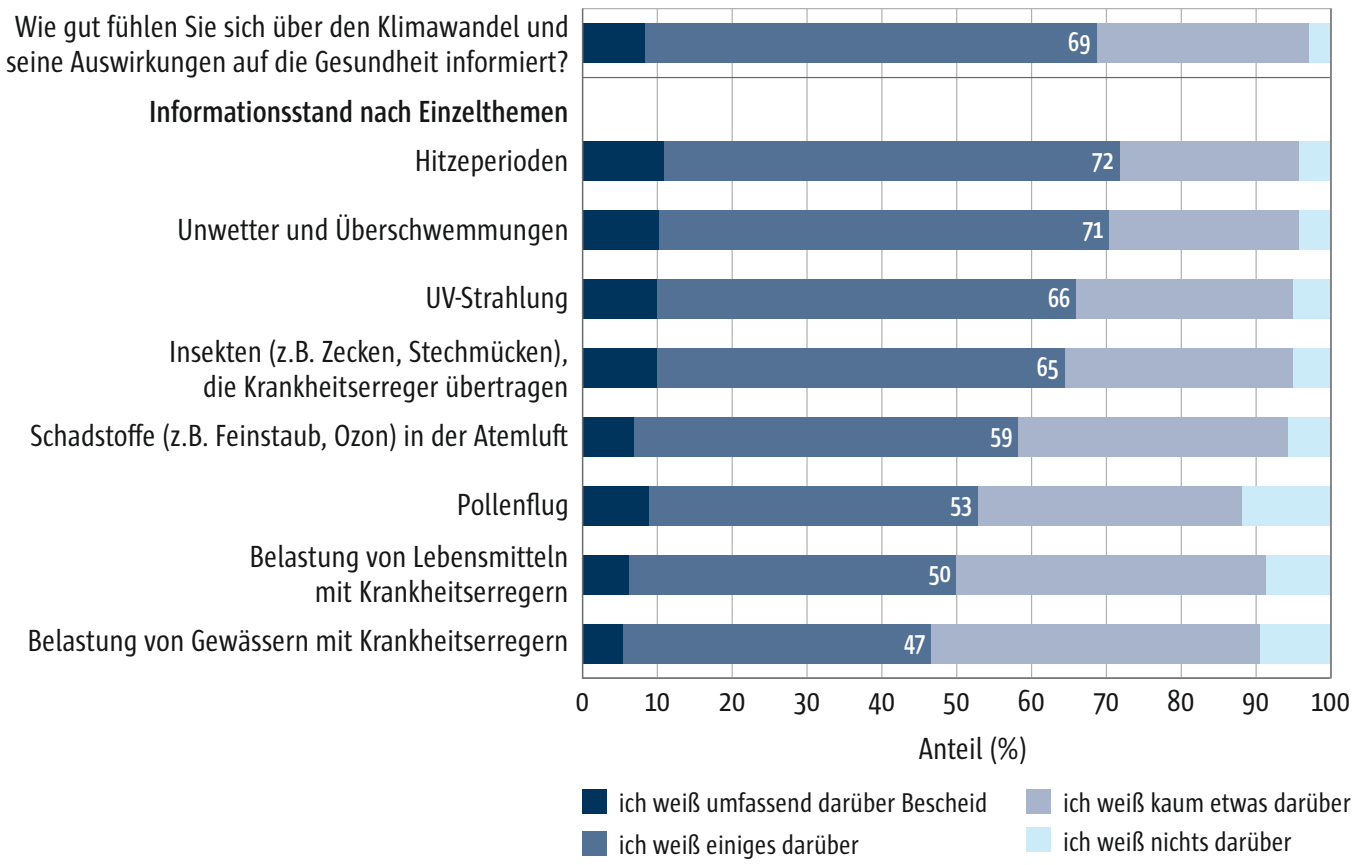

Abb. 1 Klimawandel und Gesundheit: Informationsstand in der Bevölkerung

deutsche Wohnbevölkerung. Im Vergleich zur amtlichen Statistik des Statistischen Bundesamtes weist unsere Stichprobe jedoch einen etwas höheren Anteil an Personen mit hohem Bildungsstand (35\%) auf als die Wohnbevölkerung $(29 \%)$ (vgl. Statistische Ämter der Länder und des Bundes 2019). Zudem hat die Stichprobe einen erhöhten Anteil an Personen mit gesundheitlichen Vorbelastungen. Die Hälfte der Befragten (50\%) gibt an, von mindestens einer chronischen Erkrankung betroffen zu sein. In der vom Robert Koch-Institut im Jahr 2012 durchgeführten GEDA-Studie lag dieser Anteil mit 41\% niedriger (Robert Koch-Institut 2014). Ebenso liegt der Anteil der Personen, die ihren Gesundheitszustand als sehr gut oder gut bewerten, in der vorliegenden Stichprobe (65\%) unter dem Vergleichswert der GEDA-Erhebung (70\%). Der Anteil an Personen mit deutscher Staatsangehörigkeit liegt bei $97 \%$.

\subsection{Befragungsergebnisse}

\subsubsection{Information und Risikoeinschätzung}

Um Informationsstand und Bedarf an Informationen in der Bevölkerung zu ermitteln, wurden die Teilnehmenden gefragt, wie gut sie sich insgesamt über den Klimawandel und seine Auswirkungen auf die Gesundheit informiert fühlen und wie sie ihren Informationsstand bei den verschiedenen Gesundheitsfolgen, die im $\mathrm{Zu}$ sammenhang mit dem Klimawandel diskutiert werden, einschätzen (s. Abb. 1).

Knapp 70\% der Befragten geben an, dass sie umfassend Bescheid oder zumindest einiges über die Auswirkungen des Klimawandels auf die Gesundheit wissen. Der subjektive Informationsstand hängt am deutlichsten mit dem Bildungsstand des Befragten zusammen. Je höher die Bildungsgruppe, desto höher wird der Informationsstand von den Befragten eingeschätzt ( $\mathrm{p}<0,001)$ (ohne Abbildung). 
II Gesundheitliche Auswirkungen des Klimawandels und Herausforderungen für die medizinische Versorgung in Deutschland

Tab. 2 Folgen des Klimawandels: Besorgnis in Bezug auf die eigene Gesundheit (Anteil der Antwortkategorie sehr/ziemlich in Prozent)

\begin{tabular}{|c|c|c|c|}
\hline & \multirow{2}{*}{$\begin{array}{l}\text { gesamt } \\
\text { Anteil besorgt }\end{array}$} & \multicolumn{2}{|c|}{ subjektiver Informationsstand $d^{1,2}$} \\
\hline & & $\begin{array}{l}\text { eher schlecht } \\
\text { Anteil besorgt }\end{array}$ & $\begin{array}{l}\text { eher gut } \\
\text { Anteil besorgt }\end{array}$ \\
\hline Hitzeperioden & 51,7 & 40,3 & 56,2 \\
\hline $\begin{array}{l}\text { Insekten (z.B. Zecken, Stechmücken), die Krankheitserreger } \\
\text { übertragen }\end{array}$ & 44,6 & 36,3 & 49,1 \\
\hline Unwetter und Überschwemmungen & 42,9 & 32,7 & 47,2 \\
\hline Belastung von Gewässern mit Krankheitserregern & 38,8 & 34,4 & 43,8 \\
\hline Schadstoffe (z.B. Feinstaub, Ozon) in der Atemluft & 37,1 & 32,0 & 40,6 \\
\hline Belastung von Lebensmitteln mit Krankheitserregern & 36,4 & 31,0 & 41,7 \\
\hline UV-Strahlung & 34,0 & 25,5 & 38,3 \\
\hline Pollenflug & 15,9 & 7,2 & 23,7 \\
\hline \multicolumn{4}{|c|}{$\begin{array}{l}\text { Frage: Inwieweit bereiten Ihnen die folgenden Themen Sorgen in Bezug auf Ihre eigene Gesundheit? dargestellt Antwortkategorie } \\
\text { sehr/ziemlich (in \%) } \\
{ }^{1} \text { selbst eingeschätzter Informationsstand: eher gut = ich weiß umfassend Bescheid/einiges darüber; eher schlecht = ich weiß kaum } \\
\text { etwas/nichts darüber } \\
{ }^{2} \text { jeweils signifikante Gruppenunterschiede mit } p<0,001\end{array}$} \\
\hline
\end{tabular}

Bei differenzierter Betrachtung der konkreten Gesundheitsthemen, die im Zusammenhang mit dem Klimawandel diskutiert werden, wird der Informationsbedarf deutlicher. Zwar fühlen sich knapp 70\% der Befragten gut über die (eher sichtbaren) Folgen des Klimawandels in Form von Hitzeperioden oder Unwetter und Überschwemmungen informiert. Über die möglichen gesundheitlichen Folgen einer hohen Schadstoffbelastung in der Atemluft hingegen sind nach eigener Einschätzung mehr als $40 \%$ der Befragten überhaupt nicht oder nur kaum informiert. Bei den gesundheitlichen Belastungen durch UV-Strahlung und durch von Insekten übertragbare Krankheitserreger sind es mehr als ein Drittel. Nur die Hälfte der Befragten fühlt sich gut über die gesundheitlichen Risiken des Pollenflugs (53\%) sowie durch Krankheitserreger in Lebensmitteln (50\%) und Gewässern (47\%) informiert.

\section{Mit dem Klimawandel assoziierte Gesundheits- risiken}

Um abschätzen zu können, inwieweit die hier aufgeführten Themen nicht nur zur Kenntnis genommen, sondern auch als persönliches potenzielles Gesundheitsrisiko eingeschätzt werden, wurden die Teilnehmenden gefragt, inwieweit ihnen die einzelnen Themen, die im Zusammenhang mit dem Klimawandel diskutiert werden, mit Blick auf die eigene Gesundheit Sorgen bereiten (s. Tab. 2).

Mehr als die Hälfte der Befragten (52\%) macht sich mit Blick auf die eigene Gesundheit ziemlich oder sehr Sorgen über die Zunahme an Hitzeperioden. Stärkere Besorgnis wird auch für krankheitsübertragende Insekten sowie Unwetter und Überschwemmungen geäußert, die von jeweils mehr als 40\% der Befragten als sehr/ziemlich besorgniserregend eingestuft werden. Schadstoffe in der Atemluft (37\%) und die gesundheitlichen Folgen einer erhöhten 
12 Klimawandel und Gesundheit: Welche Rolle spielt der Klimawandel im Gesundheitsbewusstsein der Befragten? Ergebnisse einer deutschlandweiten Bevölkerungsbefragung

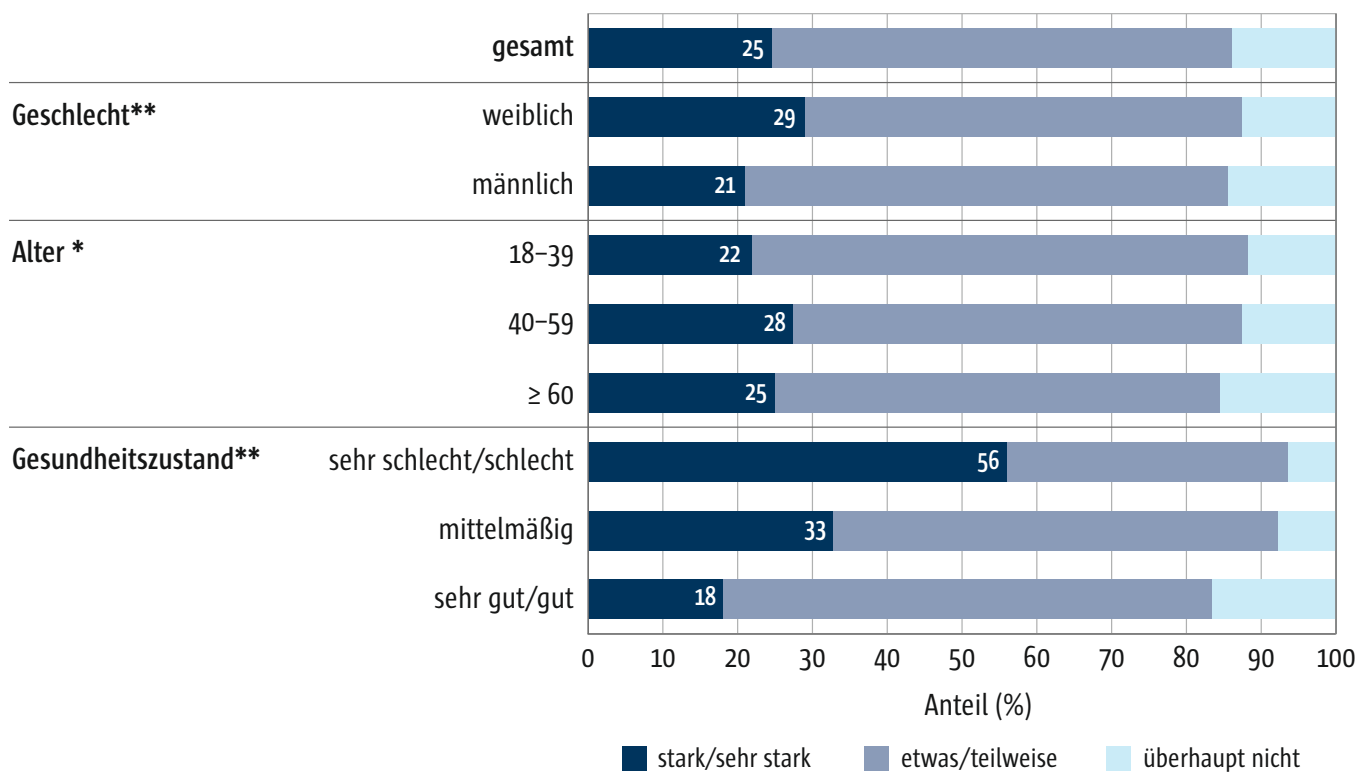

Signifikanz des Gruppenunterschiedes basierend auf Chi-Quadrat-Test nach Pearson: * $p<0,05 ;{ }^{* *} p<0,001$

Abb. 2 Subjektive Beeinträchtigung durch Hitze nach Alter, Geschlecht und Gesundheitszustand

UV-Strahlung (34\%) werden im Vergleich zu Hitzeperioden im Kontext des Klimawandels von weniger Befragten als besorgniserregend eingestuft. Ein Anteil von 16\% der Befragten äußert größere Besorgnis mit Blick auf Veränderungen der Pollenbelastung. In der gesamten Themenbreite sind Personen, die sich eher gut informiert fühlen, überdurchschnittlich häufig besorgt um die Folgen für ihre Gesundheit (signifikante Unterschiede). Dieser in einer querschnittlichen Erhebung gefundene Zusammenhang widersetzt sich allerdings einer kausalen Interpretation.

\subsubsection{Gesundheitliche Belastung und Anpassung an sich verändernde Umwelteinflüsse}

Ein weiterer Aspekt des Gesundheits- und Klimabewusstseins sind die erlebten und wahrgenommenen Beeinträchtigungen durch klimaassoziierte Ereignisse sowie die Verbreitung und Akzeptanz von geeigneten Anpassungsstrategien im Alltag. Im Folgenden werden erlebte gesundheitliche Belastungen ${ }^{1}$ und das individuelle Klimaanpassungsverhalten am Beispiel von Hitze, erhöhter UV-Strahlung, Schadstoffen in der Atemluft und Pollenbelastung untersucht.

\section{Gesundheit bei sommerlicher Hitze}

Zur Einschätzung der erlebten Hitzebelastung wurden die Teilnehmenden gefragt, wie sehr sie sich insgesamt durch anhaltende sommerliche Hitze beeinträchtigt fühlen (Skala von überhaupt nicht [1] bis sehr stark [5]) (s. Abb. 2).

1 Nach dem gängigen Belastungs-Beanspruchungskonzept von Rohmert und Rutenfranz (1975) wirken Belastungen von außen auf den Menschen ein, Beanspruchungen dagegen sind deren Auswirkungen im Individuum (einschl. unternommene Bewältigungsmaßnahmen). Demnach wären die hier genannten „subjektiven Belastungen“ als Beanspruchungen zu verstehen. 
II Gesundheitliche Auswirkungen des Klimawandels und Herausforderungen

für die medizinische Versorgung in Deutschland

Ich achte auf ausreichend Flüssigkeit zum Trinken.

Ich öffne nachts die Fenster meiner Wohnung

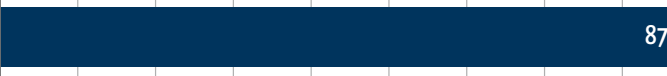

87

Ich öfne nachts die Fenster meiner Wohnung.

Ich schließe tagsüber die Gardinen, Jalousien oder Rollläden.

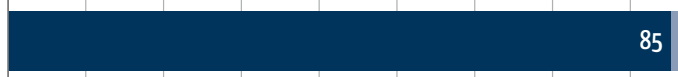

85

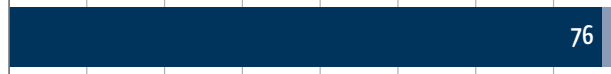

Ich meide körperliche Belastungen, auch Sport.

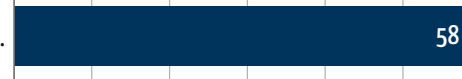

Ich beschränke meine Aktivitäten im Freien auf die Morgen- und Abendstunden.

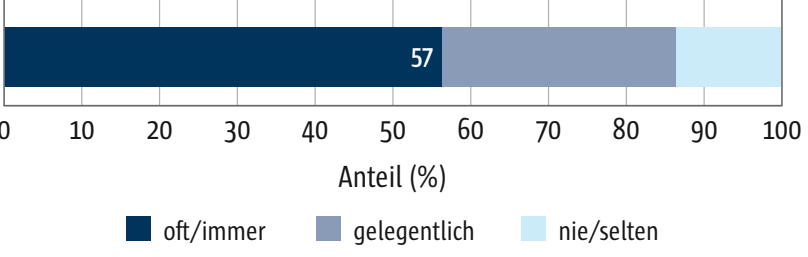

Abb. 3 Umsetzung von Verhaltensmaßnahmen zum Schutz vor Hitze $(n=3.006)$

Ein Viertel (25\%) der Befragten fühlt sich durch anhaltende Hitze stark bzw. sehr stark beeinträchtigt, 87\% der Befragten fühlen sich zumindest etwas beeinträchtigt. Die Stärke der wahrgenommenen Beeinträchtigung hängt signifikant mit dem Gesundheitszustand der Befragten zusammen. Mehr als die Hälfte der Befragten $(56 \%)$, die ihren Gesundheitszustand als schlecht/sehr schlecht bewerten, leidet unter starken Beeinträchtigungen bei Hitze, bei Personen mit gutem Gesundheitszustand sind dies dagegen weniger als ein Fünftel (18\%). Auch mit Blick auf die Altersgruppen und das Geschlecht zeigen sich signifikante Unterschiede, die aber geringer ausgeprägt sind. Personen mit chronischen Erkrankungen, wie z.B. Atemwegserkrankungen (39\%) oder Herz-Kreislauf-Erkrankungen (35\%) sind ebenfalls überdurchschnittlich häufig durch Hitze (stark/sehr stark) beeinträchtigt (krankheitsspezifische Unterschiede in der Abbildung nicht dargestellt).

Es gibt verschiedene Maßnahmen, wie Menschen ihren Tagesablauf und ihr Verhalten an Hitze anpassen können. Die aufgeführten Maßnahmen orientieren sich an den Empfehlungen des Umweltbundesamtes zum Verhalten bei sommerlicher Hitze und Hitzewellen
(UBA 2019). Das Anpassungsverhalten hinsichtlich dieser Maßnahmen wurde mit der Frage „Wie reagieren Sie im Alltag bei sommerlicher Hitze?" erhoben (Skala: nie [1], selten [2], gelegentlich [3], oft [4], und immer [5]) (s. Abb. 3).

Weit verbreitete Maßnahmen, die laut Selbstangabe oft oder immer durchgeführt werden, sind ausreichend Flüssigkeit zum Trinken (87\%), nachts die Fenster zu öffnen (85\%) und tagsüber die Wohnung abzudunkeln (76\%). Weniger Befragte vermeiden körperliche Belastungen $(58 \%)$ oder passen ihren Tagesablauf an, indem sie Aktivitäten auf die Morgen- und Abendstunden verlegen $(57 \%)$.

In der Betrachtung von Bevölkerungsgruppen (s. Tab. 3) werden die Verhaltensempfehlungen bei Hitze überwiegend in der ganzen Bevölkerung aufgegriffen, sowohl von gesundheitlich belasteten Personen (eher schlechter Gesundheitszustand) als auch von gesunden Personen (eher guter Gesundheitszustand). Ältere Menschen scheinen im Vergleich zu jüngeren häufiger ihren Tagesablauf anzupassen und Aktivitäten bei Hitze einzuschränken. Beachtenswert sind die Unterschiede im Anpassungsverhalten hinsichtlich der Informiertheit und (wesentlich deutlicher) hinsichtlich der persönli- 
12 Klimawandel und Gesundheit: Welche Rolle spielt der Klimawandel im Gesundheitsbewusstsein der Befragten? Ergebnisse einer deutschlandweiten Bevölkerungsbefragung

Tab. 3 Verhaltensmaßnahmen zum Schutz vor Hitze (Antwortkategorie oft/immer) differenziert nach Alter, Gesundheitszustand, Informiertheit und Risikoeinschätzung

\begin{tabular}{|c|c|c|c|c|c|c|c|c|c|}
\hline & & \multicolumn{2}{|c|}{ Alter in Jahren } & \multicolumn{2}{|c|}{$\begin{array}{l}\text { Gesundheits- } \\
\text { zustand }^{2}\end{array}$} & \multicolumn{2}{|c|}{$\begin{array}{l}\text { Informiertheit } \\
\text { über Hitze }\end{array}$} & \multicolumn{2}{|c|}{$\begin{array}{l}\text { Risikoeinschätzung } \\
\text { für die eigene } \\
\text { Gesundheit }^{1}\end{array}$} \\
\hline & gesamt & $\begin{array}{l}\text { unter } \\
64\end{array}$ & $\begin{array}{l}65 \text { und } \\
\text { älter }\end{array}$ & $\begin{array}{l}\text { eher } \\
\text { gut }\end{array}$ & $\begin{array}{l}\text { eher } \\
\text { schlecht }\end{array}$ & $\begin{array}{l}\text { eher } \\
\text { schlecht }\end{array}$ & $\begin{array}{l}\text { eher } \\
\text { gut }\end{array}$ & $\begin{array}{l}\text { eher } \\
\text { gering }\end{array}$ & $\begin{array}{l}\text { eher } \\
\text { hoch }\end{array}$ \\
\hline Zahl der Befragten (n) & 3.006 & 2.291 & 715 & 1.954 & 1.052 & 837 & 2.169 & 1.451 & 1.555 \\
\hline $\begin{array}{l}\text { Ich achte auf ausreichend } \\
\text { Flüssigkeit zum Trinken. }\end{array}$ & 86,7 & 85,2 & 91,3 & 87,2 & 85,7 & 80,2 & 89,2 & 82,6 & 90,5 \\
\hline $\begin{array}{l}\text { Ich öffne nachts die Fenster } \\
\text { meiner Wohnung. }\end{array}$ & 85,1 & 83,3 & 90,8 & 85,1 & 85,2 & 79,0 & 87,5 & 80,7 & 89,2 \\
\hline $\begin{array}{l}\text { Ich schließe tagsüber die } \\
\text { Gardinen, Jalousien oder } \\
\text { Rollläden. }\end{array}$ & 76,3 & 75,8 & 77,9 & 75,7 & 77,4 & 72,6 & 77,7 & 68,4 & 83,7 \\
\hline $\begin{array}{l}\text { Ich meide körperliche } \\
\text { Belastungen, auch Sport. }\end{array}$ & 58,2 & 55,0 & 68,3 & 50,6 & 72,3 & 54,5 & 59,6 & 47,0 & 68,6 \\
\hline $\begin{array}{l}\text { Ich beschränke meine } \\
\text { Aktivitäten im Freien auf die } \\
\text { Morgen-und Abendstunden. }\end{array}$ & 56,5 & 53,5 & 66,3 & 51,8 & 65,2 & 48,3 & 59,7 & 43,1 & 69,0 \\
\hline \multicolumn{10}{|c|}{$\begin{array}{l}\text { 'abgeleitet aus der Frage: Inwieweit bereiten Ihnen die folgenden Themen Sorgen in Bezug auf Ihre eigene Gesundheit? } \\
\text { eher hoch = Antwortkategorie sehr/ziemlich, eher niedrig = Antwortkategorie überhaupt nicht bis mäßig } \\
{ }^{2} \text { Gesundheitszustand eher gut = sehr gut/gut, eher schlecht = Antwortkategorien sehr schlecht/schlecht/mittel } \\
\text { : Unterschied > } 10 \text { Prozentpunkte, } \\
\text { : Unterschied > } 20 \text { Prozentpunkte }\end{array}$} \\
\hline
\end{tabular}

chen Besorgnis. Während beispielsweise knapp $70 \%$ der Befragten, die eher stark um persönliche gesundheitliche Hitzefolgen besorgt sind, körperliche Belastungen bei Hitze meiden, folgen nur 47 der Befragten, die eherwenig besorgt sind, dieser Verhaltensempfehlung. Entsprechendes gilt für die Verlagerung von Aktivitäten im Freien auf die Morgen- und Abendstunden.

\section{Gesundheit bei erhöhter UV-Strahlung}

Um das Ausmaß der wahrgenommenen Beeinträchtigungen durch UV-Strahlung zu ermitteln, wurden die Teilnehmenden gefragt, wie sehr sie sich insgesamt durch UV-Strahlung beeinträchtigt fühlen (Skala von überhaupt nicht [1] bis sehrstark [5]) (s. Abb. 4).
Eine erhöhte Sonneneinstrahlung (UVStrahlung) in Sommermonaten wird von $80 \%$ der Befragten als beeinträchtigend empfunden, von rund einem Fünftel der Befragten (19\%) sogar als stark bzw. sehr stark beeinträchtigend. Auch hier fühlen sich Personen mit sehr schlechtem/schlechtem Gesundheitszustand überdurchschnittlich häufig stark belastetet $(42 \%)$.

Ein angemessener UV-Schutz umfasst verschieden Verhaltensweisen, die in den Empfehlungen der Gesundheitsleitlinie zur Prävention von Hautkrebs (AWMF „Leitlinienprogramm Onkologie“ 2016) aufgeführt werden. Das UVSchutzverhalten der Befragten wurde mit der Frage „Wie reagieren Sie im Alltag bei starker Sonneneinstrahlung (UV-Strahlung)?" ermittelt (s. Abb. 5). 
II Gesundheitliche Auswirkungen des Klimawandels und Herausforderungen für die medizinische Versorgung in Deutschland

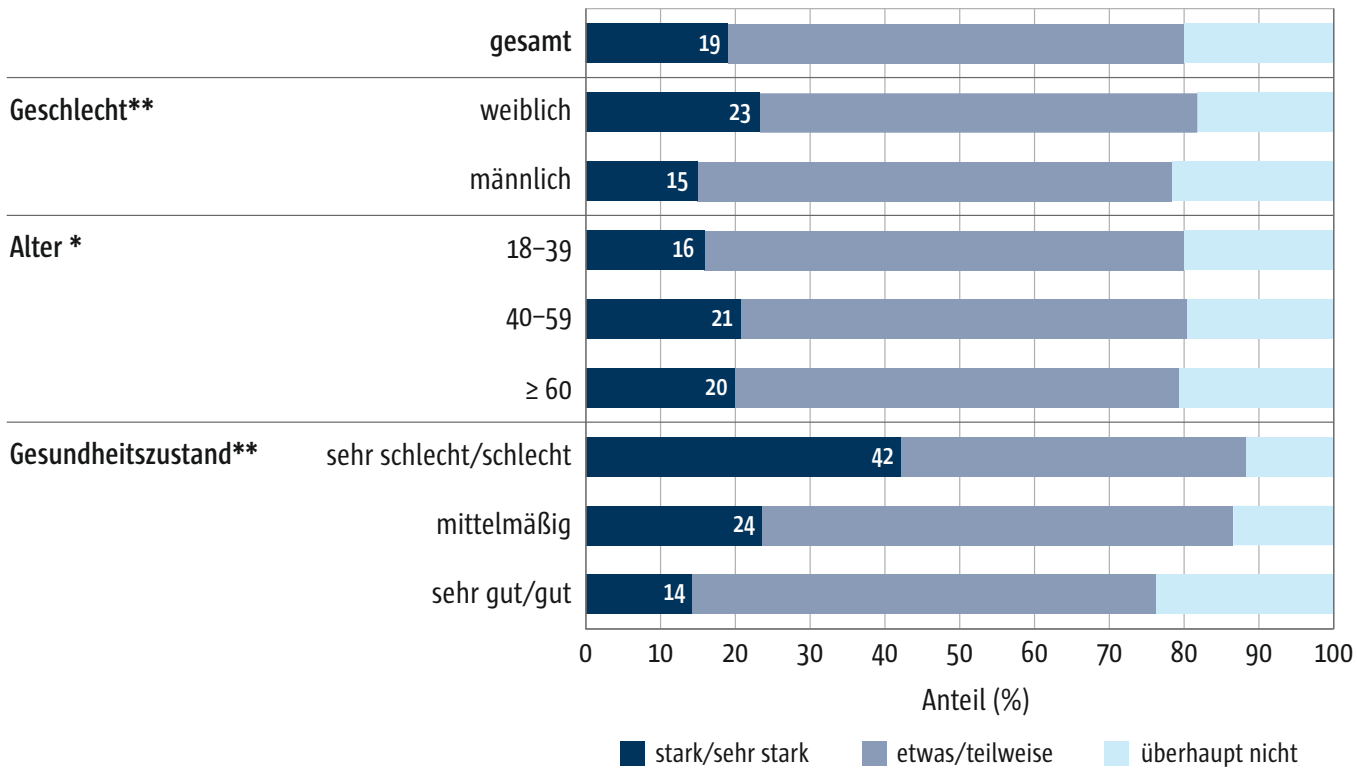

Signifikanz des Gruppenunterschiedes basierend auf Chi-Quadrat-Test nach Pearson: * $p<0,05 ;{ }^{* *} p<0,001$

Abb. 4 Subjektive Beeinträchtigung durch erhöhte Sonneneinstrahlung (UV-Strahlung) nach Alter, Geschlecht und Gesundheitszustand

Ich meide den Aufenthalt im Freien während der Mittagszeit.

Bei Aktivitäten draußen trage ich eine Sonnenbrille.

Ich benutze ein Sonnenschutzmittel (Lichtschutzfaktor mind. 20 und UVA/UVB-Schutz).

Ich trage hautbedeckende Kleidung.

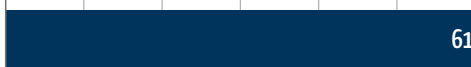

61

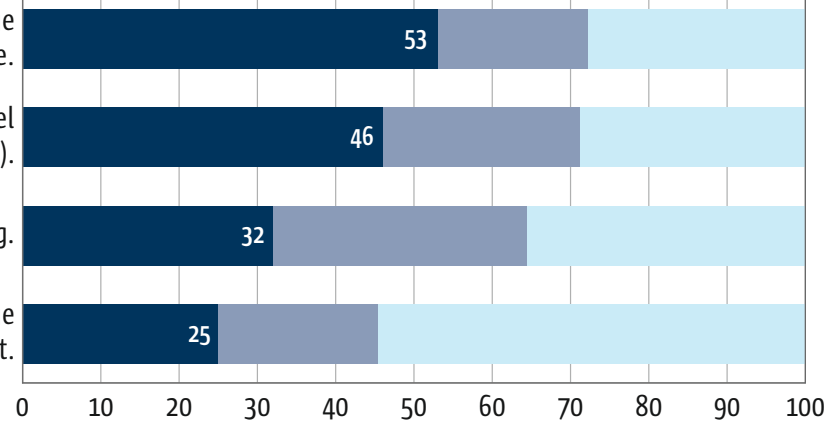

Bei Aktivitäten draußen trage ich einen Sonnenhut.

Anteil (\%)

oft/immer gelegentlich nie/selten

Abb. 5 Umsetzung von Verhaltensmaßnahmen zum Schutz vor UV-Strahlung $(n=3.006)$

Zum Schutz vor starker Sonnenstrahlung meiden $61 \%$ der Befragten (oft/immer) einen Aufenthalt im Freien während der Mittagszeit und folgen damit der vorrangigsten Maßnah- me zur Prävention von Hautkrebs. Etwas mehr als die Hälfte der Befragten (53\%) trägt (oft/immer) eine Sonnenbrille. Ein gutes Sonnenschutzmittel wird von weniger als der Hälfte 
12 Klimawandel und Gesundheit: Welche Rolle spielt der Klimawandel im Gesundheitsbewusstsein der Befragten? Ergebnisse einer deutschlandweiten Bevölkerungsbefragung

Tab. 4 Verhaltensmaßnahmen zum Schutz vor UV-Strahlung (Antwortkategorie oft/immer) differenziert nach Alter, Gesundheitszustand, Informiertheit und Risikoeinschätzung

\begin{tabular}{|c|c|c|c|c|c|c|c|c|c|}
\hline & & \multicolumn{2}{|c|}{ Alter in Jahren } & \multicolumn{2}{|c|}{$\begin{array}{l}\text { Gesundheits- } \\
\text { zustand }^{2}\end{array}$} & \multicolumn{2}{|c|}{$\begin{array}{l}\text { Informiertheit } \\
\text { über UV-Strah- } \\
\text { lung }\end{array}$} & \multicolumn{2}{|c|}{$\begin{array}{l}\text { Risikoeinschätzung } \\
\text { für die eigene } \\
\text { Gesundheit }^{1}\end{array}$} \\
\hline & gesamt & $\begin{array}{l}\text { unter } \\
64\end{array}$ & $\begin{array}{l}65 \text { und } \\
\text { älter }\end{array}$ & $\begin{array}{l}\text { eher } \\
\text { gut }\end{array}$ & $\begin{array}{l}\text { eher } \\
\text { schlecht }\end{array}$ & $\begin{array}{l}\text { eher } \\
\text { schlecht }\end{array}$ & $\begin{array}{l}\text { eher } \\
\text { gut }\end{array}$ & $\begin{array}{l}\text { eher } \\
\text { gering }\end{array}$ & $\begin{array}{l}\text { eher } \\
\text { hoch }\end{array}$ \\
\hline Zahl der Befragten (N) & 3.006 & 2.291 & 715 & 1.954 & 1.052 & 1.009 & 1.997 & 1.985 & 1.021 \\
\hline $\begin{array}{l}\text { Ich meide den Aufentalt } \\
\text { im Freien während der } \\
\text { Mittagszeit. }\end{array}$ & 61,0 & 56,8 & 74,7 & 55,1 & 72,2 & 54,7 & 64,3 & 53,2 & 76,4 \\
\hline $\begin{array}{l}\text { Bei Aktivitäten draußen } \\
\text { trage ich eine Sonnenbrille. }\end{array}$ & 52,5 & 51,0 & 57,2 & 55,0 & 47,9 & 45,6 & 56,0 & 47,4 & 62,4 \\
\hline $\begin{array}{l}\text { Ich benutze ein Sonnen- } \\
\text { schutzmittel mit Lichtschutz- } \\
\text { faktor (mind. 20) und UVA-/ } \\
\text { UVB-Schutz. }\end{array}$ & 46,1 & 46,8 & 43,8 & 48,6 & 41,5 & 36,4 & 51,0 & 36,8 & 64,3 \\
\hline $\begin{array}{l}\text { Ich trage hautbedeckende } \\
\text { Kleidung. }\end{array}$ & 32,2 & 27,8 & 46,43 & 29,3 & 37,6 & 24,5 & 36,1 & 27,5 & 41,4 \\
\hline $\begin{array}{l}\text { Bei Aktivitäten draußen } \\
\text { trage ich einen Sonnenhut. }\end{array}$ & 24,6 & 21,7 & 33,6 & 24,0 & 25,6 & 18,4 & 27,6 & 20,9 & 31,6 \\
\hline \multicolumn{10}{|c|}{$\begin{array}{l}\text { Iabgeleitet aus der Frage: Inwieweit bereiten Ihnen die folgenden Themen Sorgen in Bezug auf Ihre eigene Gesundheit? } \\
\text { eher hoch = Antwortkategorie sehr/ziemlich, eher niedrig = Antwortkategorie überhaupt nicht bis mäßig } \\
{ }^{2} \text { Gesundheitszustand eher gut = sehr gut/gut, eher schlecht = Antwortkategorien sehr schlecht/schlecht/mittel } \\
\text { : Unterschied > } 10 \text { Prozentpunkte, } \square \text { : Unterschied > } 20 \text { Prozentpunkte }\end{array}$} \\
\hline
\end{tabular}

der Bevölkerung (oft/immer) verwendet (46\%). Ein noch kleinerer Teil der Bevölkerung schützt sich oft/immer gezielt über hautbedeckende Kleidung $(32 \%)$ oder das Tragen eines Sonnenhutes (25\%).

Es gibt signifikante Unterschiede im UVSchutzverhalten der Befragten hinsichtlich Alter und Gesundheitszustand (s. Tab. 4). Menschen ab 65 Jahre bzw. mit eher schlechtem Gesundheitszustand schützen sich häufiger als jüngere bzw. gesündere Menschen, wenn sie das Haus verlassen. Besonders deutliche Unterschiede zeigen sich vor allem auch, wenn man nach der Informiertheit und der persönlichen Risikoeinschätzung für die eigene Gesundheit differenziert. Beispielsweise werden geeignete Sonnenschutzmittel von 51\% der Befragten, die sich gut über das Thema UV-Strahlung informiert fühlen, regelmäßig verwendet. Bei Personen, die sich schlecht informiert fühlen, sind es hingegen nur 36\%. Noch deutlicher ist dieser Unterschied zwischen Personen mit hoher UV-Risikoeinschätzung (64\%) und niedriger UV-Risikoeinschätzung (37\%).

\section{Gesundheit bei erhöhten Schadstoffen in der Atemluft}

Um das Ausmaß der wahrgenommenen Beeinträchtigungen durch erhöhte Schadstoffe in der Atemluft zu ermitteln, wurden die Teilnehmenden gefragt, wie sehr sie sich insgesamt durch Schadstoffe (z.B. Ozon, Feinstaub) 
II Gesundheitliche Auswirkungen des Klimawandels und Herausforderungen für die medizinische Versorgung in Deutschland

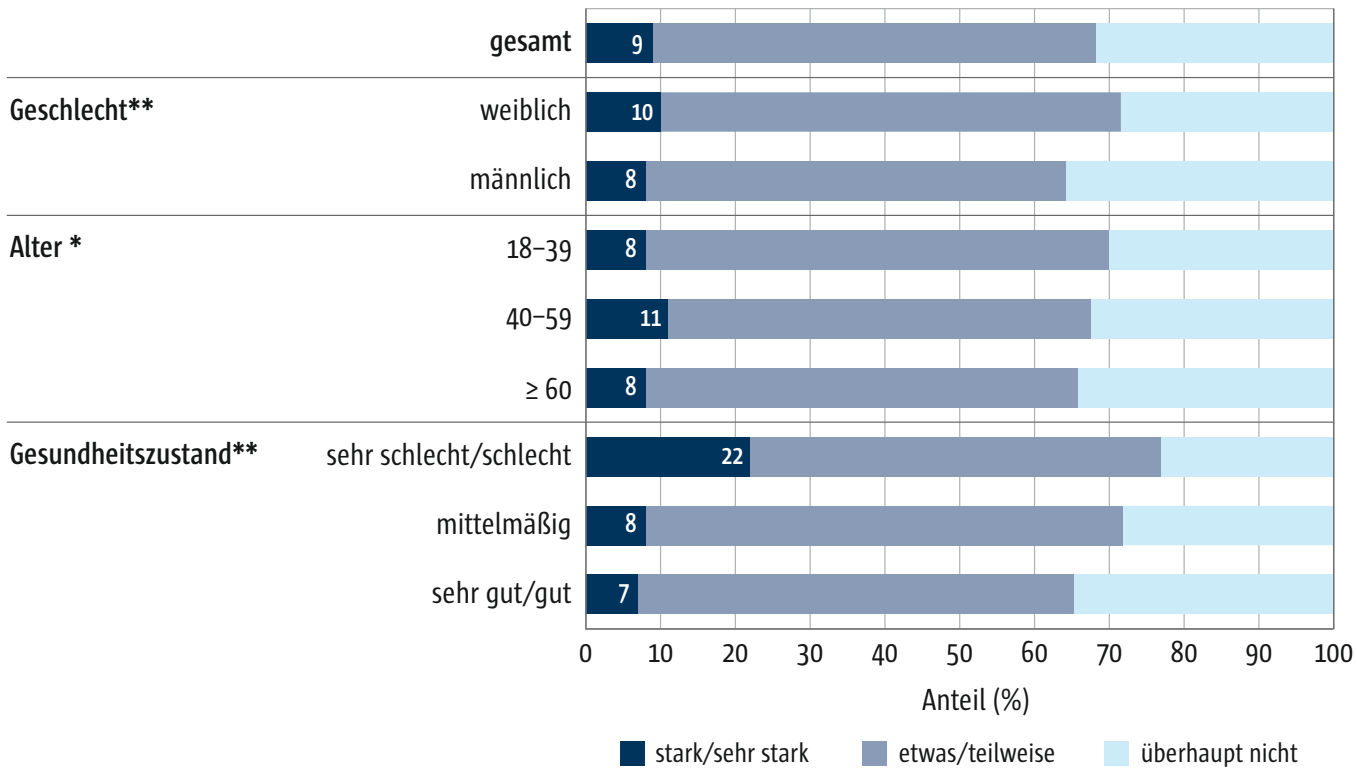

Signifikanz des Gruppenunterschiedes basierend auf Chi-Quadrat-Test nach Pearson: * $p<0,05 ;{ }^{* *} p<0,001$

Abb. 6 Subjektive Beeinträchtigung durch Schadstoffe in der Luft nach Alter, Geschlecht und Gesundheitszustand

beeinträchtigt fühlen (Skala von überhaupt nicht [1] bis sehr stark [5]) (s. Abb. 6).

Schadstoffe in der Atemluft werden etwa von zwei Dritteln der Befragten (68\%) als beeinträchtigend empfunden, allerdings von nur einem Zehntel der Befragten ( $9 \%$ ) als stark oder sehr stark beeinträchtigend erlebt. Auch Luftschadstoffe beeinträchtigen überdurchschnittlich häufig Personen mit schlechterem Gesundheitszustand stark/sehr stark (22\%). Unter den ausgewerteten Indikationen fiel vor allem die Bevölkerungsgruppe mit Atemwegserkrankungen auf, die mit $17 \%$ ebenfalls überdurchschnittlich häufig stärkere Beeinträchtigungen berichtet.

Teilnehmende wurden auch gefragt, wie sie reagieren, wenn sie die Luftqualität als schlecht empfinden. Dafür wurden drei Verhaltensmaßnahmen aufgeführt, die sich an den Empfehlungen des UBA zum Verhalten bei erhöhten Schadstoffkonzentrationen (Ozon) orientieren
(UBA 2019). Die häufigsten Reaktionen der Befragten auf schlechte Luftqualität (Antwortkategorie oft/immer) sind körperliche Belastungen, wie Sport, zu meiden (29\%) oder die Fenster geschlossen zu halten (28\%). Rund ein Fünftel der Befragten (18\%) reduziert insgesamt den Aufenthalt im Freien (s. Abb. 7).

Individuelle Anpassungsmaßnahmen an schlechte Luftqualität sind in der Bevölkerung erkennbar weniger weit verbreitet als Maßnahmen zum Schutz vor Hitze und UV-Strahlung. Ältere Menschen und gesundheitlich belastete Personen reagieren deutlich häufiger mit Einschränkung ihrer Aktivitäten im Freien (s. Tab. 5). Personen, die das Risiko von Luftschadstoffen für ihre eigene Gesundheit als eher hoch einstufen, setzen schützende Verhaltensmaßnahmen deutlich häufiger um als Personen, die ihr gesundheitliches Risiko eher niedrig einstufen. 
12 Klimawandel und Gesundheit: Welche Rolle spielt der Klimawandel im Gesundheitsbewusstsein der Befragten? Ergebnisse einer deutschlandweiten Bevölkerungsbefragung

Ich meide körperliche Belastung im Freien, auch Sport.

Ich halte die Fenster geschlossen

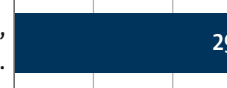

29

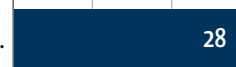

Ich reduziere den Aufenthalt im Freien.

18

Ich verwende Arzneimittel gegen die Symptome.

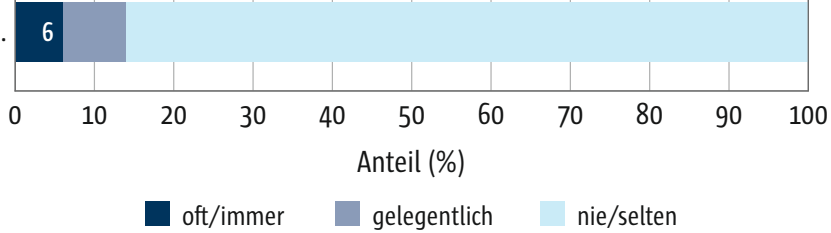

Abb. 7 Umsetzung von Verhaltensmaßnahmen bei erhöhter Schadstoffbelastung in der Luft $(n=3.006)$

Tab. 5 Verhalten bei erhöhter Schadstoffbelastung in der Luft (Antwortkategorie oft/immer) differenziert nach Alter, Gesundheitszustand, Informiertheit und Risikoeinschätzung

\begin{tabular}{|c|c|c|c|c|c|c|c|c|c|}
\hline & & \multicolumn{2}{|c|}{ Alter in Jahren } & \multicolumn{2}{|c|}{$\begin{array}{l}\text { Gesundheits- } \\
\text { zustand }{ }^{2}\end{array}$} & \multicolumn{2}{|c|}{$\begin{array}{l}\text { Informiertheit } \\
\text { über Schadstoffe }\end{array}$} & \multicolumn{2}{|c|}{$\begin{array}{l}\text { Risikoeinschätzung } \\
\text { für die eigene } \\
\text { Gesundheit }^{1}\end{array}$} \\
\hline & gesamt & $\begin{array}{l}\text { unter } \\
64\end{array}$ & $\begin{array}{l}65 \text { und } \\
\text { älter }\end{array}$ & $\begin{array}{l}\text { eher } \\
\text { gut }\end{array}$ & $\begin{array}{l}\text { eher } \\
\text { schlecht }\end{array}$ & $\begin{array}{l}\text { eher } \\
\text { schlecht }\end{array}$ & $\begin{array}{l}\text { eher } \\
\text { gut }\end{array}$ & $\begin{array}{l}\text { eher } \\
\text { gering }\end{array}$ & $\begin{array}{l}\text { eher } \\
\text { hoch }\end{array}$ \\
\hline Zahl der Befragten (N) & 3.006 & 2.291 & 715 & 1.954 & 1.052 & 1.236 & 1.770 & 1.891 & 1.115 \\
\hline $\begin{array}{l}\text { Ich meide körperliche } \\
\text { Belastungen im Freien, } \\
\text { auch Sport. }\end{array}$ & $28,9 \%$ & 24,5 & 43,2 & 21,3 & 43,2 & 24,2 & 32,3 & 19,9 & 44,3 \\
\hline $\begin{array}{l}\text { Ich halte die Fenster } \\
\text { geschlossen. }\end{array}$ & $28,1 \%$ & 25,3 & 37,3 & 24,9 & 34,2 & 23,1 & 31,7 & 19,9 & 42,2 \\
\hline $\begin{array}{l}\text { Ich reduziere den Aufenthalt } \\
\text { im Freien. }\end{array}$ & $17,7 \%$ & 15,0 & 26,3 & 13,1 & 26,4 & 14,1 & 20,3 & 9,5 & 31,8 \\
\hline $\begin{array}{l}\text { Ich verwende Arzneimittel } \\
\text { gegen die Symptome. }\end{array}$ & $5,8 \%$ & 5,5 & 6,4 & 3,8 & 9,4 & 4,5 & 6,6 & 2,9 & 10,7 \\
\hline $\begin{array}{l}\text { 'abgeleitet aus der Frage: Inwiewe } \\
\text { eher hoch = Antwortkategorie sehr } \\
{ }^{2} \text { Gesundheitszustand eher gut }=\text { se } \\
\text { : Unterschied }>10 \text { Prozentpunt }\end{array}$ & $\begin{array}{l}\text { bereite } \\
\text { ziemlich } \\
\text { gut/gu } \\
\text { : Un }\end{array}$ & $\begin{array}{l}\text { nen die } \\
\text { r nied } \\
\text { er sch } \\
\text { hied > }\end{array}$ & $\begin{array}{l}\text { enden } \\
\text { Antwor } \\
=\text { Antw } \\
\text { ozentp }\end{array}$ & kategc & en in $\mathrm{E}$ & $\begin{array}{l}\text { uf lhre } \\
\text { t bis ma } \\
\text { t/schle }\end{array}$ & ittel & & \\
\hline
\end{tabular}

\section{Gesundheit bei erhöhter Pollenbelastung}

Zur Ermittlung der erlebten Beeinträchtigungen durch Pollen wurden die Teilnehmenden gefragt, wie sehr sie sich insgesamt durch Pol- len beeinträchtigt fühlen (Skala von überhaupt nicht [1] bis sehr stark [5]) (s. Abb. 8).

Insgesamt fühlen sich $45 \%$ der Befragten in irgendeiner Weise von gesundheitlichen Beeinträchtigungen durch Pollen betroffen, mehr als 


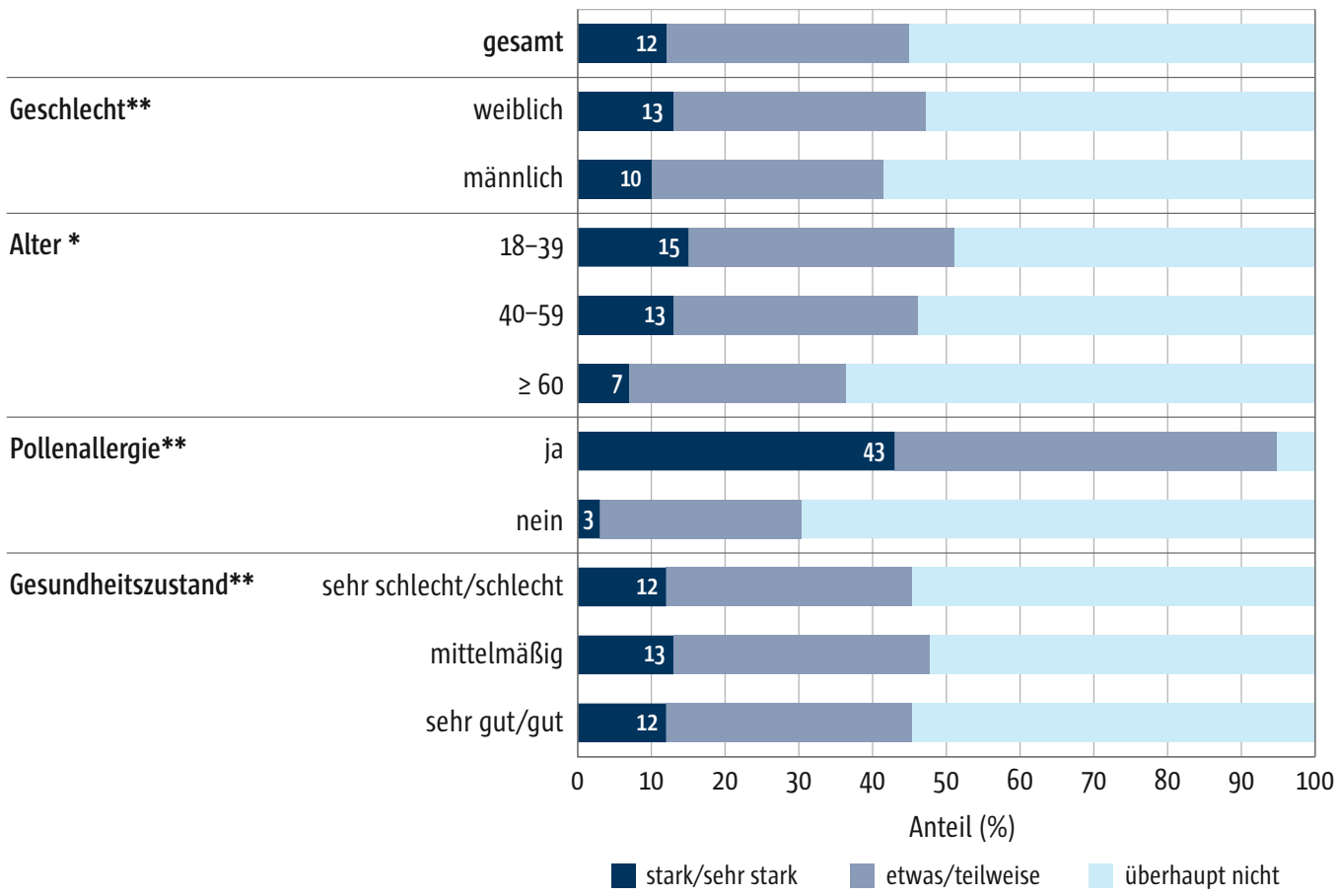

Signifikanz des Gruppenunterschiedes basierend auf Chi-Quadrat-Test nach Pearson: * $p<0,05 ;{ }^{* *} p<0,001$

Abb. 8 Subjektive Beeinträchtigung durch Pollen nach Alter, Geschlecht und Gesundheitszustand

ein Zehntel (12\%) stark bzw. sehr stark. Jüngere Menschen im Alter von 18 bis 39 Jahren berichten mehr als doppelt so häufig stärkere Beeinträchtigungen ( $15 \%$ ) als Personen über 6o Jahre (7\%). Personen mit Pollenallergie (43\%) und Personen mit Asthma (31\%) geben erwartungsgemäß besonders häufig an, von starken/sehr starken Beeinträchtigungen betroffen zu sein. Der generelle subjektive Gesundheitszustand des Befragten steht in keinem signifikanten Zusammenhang mit Pollenbeschwerden.

Zur Untersuchung des Schutzverhaltens wurden die Teilnehmenden gefragt, welche präventiven Maßnahmen sie in Zeiten des Pollenflugs zum Schutz vor Pollenbeschwerden anwenden (entsprechende Maßnahmen sind z.B. in Kap. 10 aufgeführt). Hierfür werden Personen betrachtet, die sich durch Pollen subjektiv beeinträchtigt fühlen (daher auch Personen ohne ärztlich diagnostizierte Pollenallergie [45\%, s. Abb. 8]).
Die aufgeführten Schutzmaßnahmen werden auch von Personen mit pollenallergischen Beschwerden nur begrenzt umgesetzt (s. Abb. 9). Weniger als ein Drittel der Betroffenen setzt die Verhaltensempfehlungen bei Pollenflug regelmäßig um. So halten 33\% der Betroffenen (oft/immer) zum Schutz vor Beschwerden „die Fenster geschlossen“, 30\% „waschen abends die Haare " und 29\% der Befragten „vermeiden Ausflüge auf Wiesen und Felder“. Ein weiteres Viertel der Befragten führt die Verhaltensmaßnahmen zumindest gelegentlich durch. Etwa die Hälfte der Betroffenen (46\%) verwenden (zumindest gelegentlich) Arzneimittel gegen die Symptome (z.B. Antiallergi$\mathrm{ka}$ ). Auffallend sind auch hier die Unterschiede hinsichtlich Informiertheit bzw. persönlicher Besorgnis und dem Schutzverhalten (s. Tab. 6). Personen mit besserer Informiertheit und höherer Besorgnis setzen wesentlich häufiger die 
12 Klimawandel und Gesundheit: Welche Rolle spielt der Klimawandel im Gesundheitsbewusstsein der Befragten? Ergebnisse einer deutschlandweiten Bevölkerungsbefragung

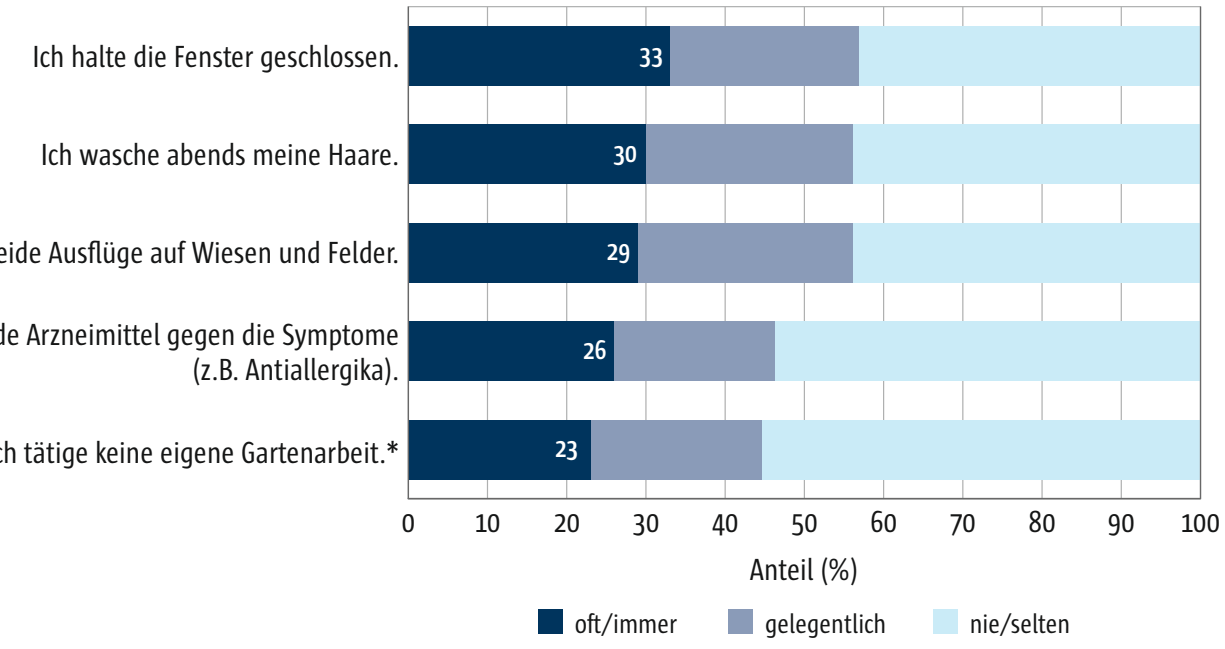

Abb. 9 Umsetzung von Verhaltensmaßnahmen bei erhöhter Pollenbelastung $(n=1.345$, Personen mit pollenallergischen Beschwerden, *abweichend Grundgesamtheit: zutreffende Angaben $n=992$ )

Tab. 6 Verhalten bei erhöhter Pollenbelastung (Antwortkategorie oft/immer) von Personen mit Pollenallergie bzw. -beschwerden ( $n=1.345$ ) differenziert nach Alter, Gesundheitszustand, Informiertheit und Risikoeinschätzung

\begin{tabular}{|c|c|c|c|c|c|c|c|c|c|}
\hline & & \multicolumn{2}{|c|}{ Alter in Jahren } & \multicolumn{2}{|c|}{$\begin{array}{l}\text { Gesundheits- } \\
\text { zustand }^{2}\end{array}$} & \multicolumn{2}{|c|}{$\begin{array}{l}\text { Informiertheit } \\
\text { über Pollen }\end{array}$} & \multicolumn{2}{|c|}{$\begin{array}{l}\text { Risikoeinschätzung } \\
\text { für die eigene } \\
\text { Gesundheit }^{1}\end{array}$} \\
\hline & gesamt & $\begin{array}{l}\text { unter } \\
64\end{array}$ & $\begin{array}{l}65 \text { und } \\
\text { älter }\end{array}$ & $\begin{array}{l}\text { eher } \\
\text { gut }\end{array}$ & $\begin{array}{l}\text { eher } \\
\text { schlecht }\end{array}$ & $\begin{array}{l}\text { eher } \\
\text { schlecht }\end{array}$ & $\begin{array}{l}\text { eher } \\
\text { gut }\end{array}$ & $\begin{array}{l}\text { eher } \\
\text { gering }\end{array}$ & $\begin{array}{l}\text { eher } \\
\text { hoch }\end{array}$ \\
\hline Zahl der Befragten (N) & 1.345 & 1.091 & 254 & 846 & 499 & 449 & 896 & 926 & 419 \\
\hline $\begin{array}{l}\text { Ich halte die Fenster } \\
\text { geschlossen. }\end{array}$ & 33,4 & 31,7 & 40,6 & 32,7 & 34,5 & 24,3 & 38,0 & 24,2 & 53,7 \\
\hline $\begin{array}{l}\text { Ich wasche abends meine } \\
\text { Haare. }\end{array}$ & 30,3 & 33,1 & 18,5 & 33,6 & 24,9 & 24,3 & 33,4 & 24,5 & 43,2 \\
\hline $\begin{array}{l}\text { Ich vermeide Ausflüge auf } \\
\text { Wiesen und Felder. }\end{array}$ & 29,4 & 27,9 & 36,2 & 26,6 & 34,3 & 17,8 & 35,3 & 17,5 & 55,9 \\
\hline $\begin{array}{l}\text { Ich verwende Arzneimittel } \\
\text { gegen die Symptome } \\
\text { (z.B. Antiallergika). }\end{array}$ & 26,1 & 27,4 & 20,5 & 27,0 & 24,7 & 15,8 & 31,3 & 15,6 & 49,4 \\
\hline $\begin{array}{l}\text { Ich tätige keine eigene } \\
\text { Gartenarbeit.* }\end{array}$ & 22,9 & 22,5 & 25,2 & 21,1 & 26,1 & 22,1 & 23,3 & 17,9 & 34,3 \\
\hline \multicolumn{10}{|c|}{$\begin{array}{l}\text { ªbgeleitet aus der Frage: Inwieweit bereiten Ihnen die folgenden Themen Sorgen in Bezug auf Ihre eigene Gesundheit? } \\
\text { eher hoch = Antwortkategorie sehr/ziemlich, eher niedrig = Antwortkategorie überhaupt nicht bis mäßig } \\
{ }^{2} \text { Gesundheitszustand eher gut = sehr gut/gut, eher schlecht = Antwortkategorien sehr schlecht/schlecht/mittel } \\
\text { : Unterschied > } 10 \text { Prozentpunkte, } \square \text { : Unterschied > } 20 \text { Prozentpunkte } \\
\text { *abweichend Grundgesamtheit: zutreffende Angaben n = } 992\end{array}$} \\
\hline
\end{tabular}


II Gesundheitliche Auswirkungen des Klimawandels und Herausforderungen für die medizinische Versorgung in Deutschland

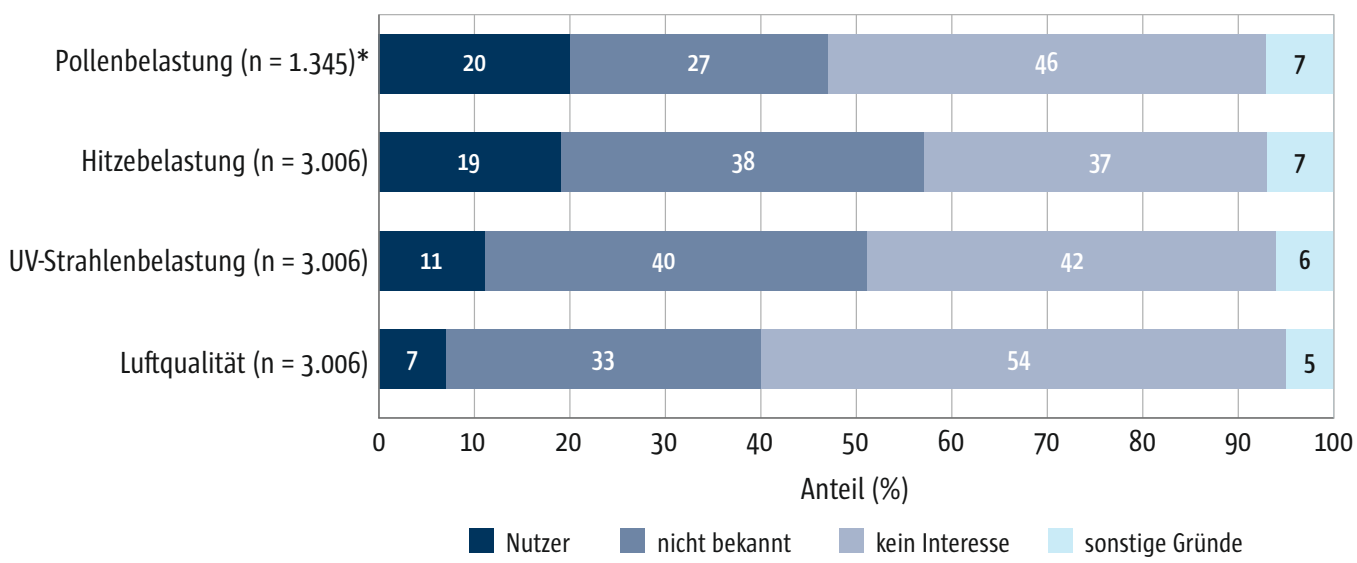

*Personen mit Pollenbeschwerden (auch leichten, Kategorie „etwas“ bis „sehr stark“)

Abb. 10 Nutzung von Warn- und Informationsdiensten zu Umweltbelastungen

Verhaltensempfehlungen für Pollenallergiker um als Personen, bei denen Informationsstand und Besorgnis weniger stark ausgeprägt sind.

\subsubsection{Nutzung von Informationsdiensten und Vorhersagen zu Umwelt- belastungen}

Vorhersagen und Warnungen zu Umweltereignissen (z.B. Hitzewarnungen) sind ein weiterer Aspekt der Verhaltensprävention. Der Deutsche Wetterdienst (DWD) bietet tagesaktuelle Vorhersagen und Warnungen zu gefährdenden Wetterlagen, UV-Warnungen und Pollenflugvorhersagen an. Das Umweltbundesamt (UBA) veröffentlicht den Luftqualitätsindex, der aus den gemessenen Konzentrationen von Stickstoffdioxid, Feinstaub $\left(\mathrm{PM}_{10}\right)$ und Ozon berechnet wird. Diese Informationsdienste richten sich an die Bevölkerung und haben das Ziel, zur Durchführung von Schutz- oder Anpassungsmaßnahmen zu motivieren (Capellaro u. Sturm 2015).

Um Bekanntheit und Interesse an Warnund Informationsdiensten in der Bevölkerung abschätzen zu können, wurden die Teilnehmenden gefragt, ob sie einen konkreten Warn- oder Informationsdienst zu den hier untersuchten Umweltbelastungen nutzen (s. Abb. 10).

Warn- und Informationsdienste werden noch relativ selten genutzt und sind vielen der Befragten auch nicht bekannt. Am häufigsten genutzt wird der Pollenfluginformationsdienst, den etwa ein Fünftel der Zielgruppe (Personen mit Pollenbeschwerden) nutzen (20\%). Informationsdienste $\mathrm{zu}$ Hitzebelastungen werden von weniger als einem Fünftel der Befragten genutzt (19\%) und sind mehr als einem Drittel der Befragten auch nicht bekannt (38\%). Nur etwa ein Zehntel der Befragten nutzt Informationsdienste zur UV-Strahlenbelastung ( $11 \%)$ oder Luftqualität ( $7 \%)$. Auffällig ist, dass insbesondere Informationsdienste zur Luftqualität bei den Befragten kein Interesse finden (54\%).

\subsection{Zusammenfassende Diskussion}

Die Auswirkungen des Klimawandels erfordern konkrete und zielgerichtete Anpassungsstrategien im Bereich der Gesundheitsvorsorge und -prävention (Bundesregierung 2020). Neben Umwelt- und Klimaschutzmaßnahmen (die hier nicht thematisiert werden) müssen in der Bevölkerung verstärkt präventive Verhaltens- 
maßnahmen zum Schutz vor den gesundheitlichen Folgen des Klimawandels etabliert werden (Bund/Länder Ad-hoc-Arbeitsgruppe „Gesundheitliche Anpassung an die Folgen des Klimawandels [GAK]“ 2017).

Die Befragungsergebnisse sprechen dafür, dass weite Teile der Bevölkerung noch nicht ausreichend über die gesundheitlichen Auswirkungen des Klimawandels informiert sind. Bei direkt erlebbaren Umweltereignissen wie Hitze oder Unwettern signalisiert immerhin ein Drittel der Befragten klaren Informationsbedarf. Noch deutlicher zeigen sich Informationsdefizite bei Umweltrisiken, die im Zusammenhang mit dem Klimawandel noch vergleichsweise selten Beachtung finden, wie erhöhte Belastungen durch Luftverschmutzung, Pollenallergene oder durch Wasser und Lebensmittel übertragene Krankheitserreger (s. Abb. 1). 40 bis 50\% der Befragten geben an, über diese Themenfelder nicht gut informiert zu sein.

Mit Blick auf die eigene Gesundheit äußern die Befragten in besonderem Maß Besorgnis über Klimafolgen, die durch zunehmende Extremwetterereignisse (Hitze und Unwetter) hervorgerufen werden. Die weniger erlebbaren Folgen des Klimawandels, wie erhöhte Schadstoffbelastungen in der Atemluft, Strahlen- oder Pollenbelastungen, werden in der Gesamtbevölkerung hingegen deutlich seltener als besorgniserregend für die eigene Gesundheit wahrgenommen, obwohl sie aus umweltmedizinischer und epidemiologischer Sicht nicht weniger gesundheitsrelevant sind (s. Kap. 8, 9, 10). Zu vergleichbaren Ergebnissen (mit etwas veränderter Frageformulierung) kam auch eine Studie des Bundesinstituts für Risikobewertung (BfR) zur Wahrnehmung des Klimawandels (Berger et al. 2019). Auch in dieser Studie lösten indirekte und weniger sichtbare Folgen des Klimawandels weniger Beunruhigung unter den Befragten aus und sollten in der Konsequenz daher stärker bei der Risikokommunikation berücksichtigt werden. Auffallend in der vorliegenden WIdO-Befragung ist, dass ein großer Teil der Befragten (45\%) sich auch Sorgen um die Ausbreitung von krank- heitsübertragenden Insekten macht (s. Tab. 2). Möglicherweise ist das auf eine erhöhte Medienaufmerksamkeit für klimasensitive Zecken zurückzuführen, die in Deutschland weit verbreitet sind und zu den Überträgern von Infektionen (wie FSME und Borreliose) zählen (s. Kap. 11). Ferner wird in den Medien im Zusammenhang mit dem Klimawandel auch verstärkt über die Ausbreitung von invasiven Mückenarten berichtet, wie im Fall des West-Nil-Fiebers, das im Jahr 2019 zum ersten Mal in Deutschland durch Mücken übertragen wurde (vgl. RKI 2019). Die Ergebnisse der aktuellen Befragung bestärken den Bedarf an fundierten themenspezifischen Informationen, die die Öffentlichkeit erreichen und das breite Themenfeld der klimarelevanten Gesundheitsrisiken abdecken.

Eine zentrale Frage dieser Studie war, in welchem Umfang sich die Befragten im Alltag vor klimaassoziierten Umweltbelastungen schützen. Die Ergebnisse lassen deutliche Unterschiede im Umgang mit den hier untersuchten Umweltrisiken erkennen. Verhaltensempfehlungen zum Schutz vor Hitze finden bei weiten Teilen der Befragten Zustimmung. Deutlich weniger verbreitet sind Verhaltensmaßnahmen zum Schutz vor UV-Strahlung, erhöhten Schadstoffkonzentrationen oder Pollenbelastung. Eine mögliche Erklärung hierfür ist, dass diese Umweltrisiken weit weniger als Hitze subjektiv durch den Menschen wahrnehmbar sind und gesundheitlichen Beeinträchtigungen zugeordnet werden können. In der Befragung wird das besonders deutlich am Beispiel der Luftschadstoffe: Weniger als ein Zehntel der Befragten fühlt sich durch erhöhte Schadstoffe in der Atemluft stark beeinträchtigt, bei Hitze sind es mehr als ein Viertel der Befragten, obgleich beide Umweltfaktoren (insbesondere in Städten) häufig zusammen auftreten (s. Kap. 8). Insgesamt legen die Ergebnisse aus dieser Befragung nahe, dass gesundheitsförderliche Maßnahmen zum Schutz vor Umweltrisiken in der Bevölkerung noch nicht ausreichend etabliert sind. Offensichtlich werden insbesondere Risiken von Umweltfaktoren 
II Gesundheitliche Auswirkungen des Klimawandels und Herausforderungen

für die medizinische Versorgung in Deutschland

(wie beispielsweise Luftschadstoffen oder UVStrahlung), deren Zusammenhang mit subjektiven Auswirkungen nicht unmittelbar erkennbar ist, eher unterschätzt.

Unter den Befragten zeigen sich auch erhebliche Unterschiede im Schutzverhalten. Ältere Menschen und Menschen mit schlechterem Gesundheitszustand passen ihr Verhalten häufiger an erhöhte Umweltbelastungen an als jüngere und gesunde Menschen. Themenübergreifend war zudem festzustellen, dass Personen mit besserem Informationsstand und stärkerer Besorgnis für die gesundheitlichen Folgen des Klimawandels wesentlich häufiger mit Schutzmaßnahmen reagieren als Personen, bei denen Informiertheit und Risikoeinschätzung geringer ausgeprägt waren. Auch dieser Befund liefert einen klaren Anhaltspunkt für stärkere Bemühungen um Information und Aufklärung in der Bevölkerung. Allerdings ist das Klimaanpassungs- und Schutzverhalten ein komplexer Prozess, der von verschiedenen Faktoren (z.B. auch eingeschränkten Ressourcen, Umwelteinstellungen) beeinflusst wird. Um valide Einflussfaktoren auf das Anpassungsverhalten identifizieren zu können, bedarf es verbesserter Analysen auf Basis verhaltenswissenschaftlicher Erklärungsansätze (ausführlicher hierzu s. Kap. 13).

Übereinstimmend mit anderen Studien (Kunz-Plapp et al. 2016; Borchers et al. 2020) zeigt sich auch in dieser Befragung, dass Personen mit schlechtem Gesundheitszustand wesentlich stärker durch Umweltbelastungen beeinträchtigt sind als gesunde Personen. Dies dürfte sich dadurch erklären, dass der Organismus von gesundheitlich belasteten Personen nicht in gleicher Weise auf extreme Situationen reagieren kann, wie es bei Gesunden der Fall ist, und dass zudem chronisch Erkrankte häufig mit einer Verschlechterung ihrer Symptomatik reagieren (Bund/Länder Ad-hoc-Arbeitsgruppe „Gesundheitliche Anpassung an die Folgen des Klimawandels [GAK]“ 2017). Daher müssen sich Informationen zu Schutzmaßnahmen im besonderen Maße auch an vulnerable Bevölkerungsgruppen richten.
Die Anpassung und Weiterentwicklung von Informations- und Frühwarnsystemen ist schon Teil der Deutschen Anpassungsstrategie an den Klimawandel im Handlungsfeld Menschliche Gesundheit (Bundesregierung 2020). Informationen über zu erwartende Umweltbelastungen können von verschiedenen Stellen tagesaktuell bezogen werden. Sie sollen über institutionalisierte Kommunikationswege (z.B. Landesämter, Gesundheitsämter) und nicht-institutionalisierte Informationswege (Radio, TV, Presse und Internet) die Öffentlichkeit erreichen (Capellaro u. Sturm 2015). Die Ergebnisse aus dieser Umfrage zeigen, dass bestehende Warn-und Informationsdienste weiten Teilen der Bevölkerung nicht bekannt sind bzw. kein Interesse besteht. Die Nutzung ist noch sehr schwach ausgeprägt.

Letztlich zeigen die Ergebnisse, dass weiter deutlicher Informationsbedarf $\mathrm{zu}$ den gesundheitlichen Risiken des Klimawandels in der Gesellschaft besteht. Das Schutzverhalten in der Bevölkerung ist verbesserungsfähig, gerade auch mit Blick auf ältere Menschen und vulnerable Gruppen, die besonders gefährdet sind. Vor dem Hintergrund des weiteren Fortschreitens des Klimawandels scheint dies umso mehr geboten. Es besteht eine erhebliche kommunikative Herausforderung auf dem Weg, das individuelle präventive Verhalten zu stärken, wobei auch die heute vorhandenen Informations- und Frühwarnsysteme noch deutlich intensiver genutzt werden können. Verstärkt treten die kommunikativen Herausforderungen bei klimabedingten Gesundheitsbelastung zu Tage, die von den Menschen nicht unmittelbar erlebt werden, aber deswegen keineswegs weniger gefährlich sind.

\section{Literatur}

AWMF „Leitlinienprogramm Onkologie“ (2016) Prävention von Hautkrebs. Gesundheitsleitlinie. URL: https://www.awmf. org/uploads/tx_szleitlinien/032-0520L__Pr\%C3\%A4vention_ von_Hautkrebs_2014-04.pdf (abgerufen am 27.02.2021)

Berger N, Lindemann AK, Böl GB (2019) Wahrnehmung des Klimawandels durch die Bevölkerung und Konsequenzen für die Risikokommunikation. Bundesgesundheitsbl 62:612-619 
Borchers P, Looks P, Reinfried F, Oertel H, Kugler I (2020) Subjektive Hitzebelastung in einzelnen Fokusgebieten Dresdens. Eine Untersuchung klimatischer, stadtstruktureller und sozialer Merkmale zur Ermittlung von Risikogebieten und Risikogruppen. Präv Gesundheitsf 15:303-309

Bund/Länder Ad-hoc-Arbeitsgruppe ,Gesundheitliche Anpassung an die Folgen des Klimawandels (GAK) ' (2017) Handlungsempfehlungen für die Erstellung von Hitzeaktionsplänen zum Schutz der menschlichen Gesundheit. Bundesgesundheitsbl 60:662-672

Bundesregierung (2020) Zweiter Fortschrittsbericht zur Deutschen Anpassungsstrategie an den Klimawandel. URL: https://www. bmu.de/fileadmin/Daten_BMU/Download_PDF/Klimaschutz/ klimawandel_das_2_fortschrittsbericht_bf.pdf (abgerufen am 27.02.2021)

Capellaro M, Sturm D (2015) Evaluation von Informationssystemen zu Klimawandel und Gesundheit. Umweltbundesamt, Dessau-Roßlau (Band 1: Anpassung an den Klimawandel: Evaluation bestehender nationaler Informationssysteme [UVIndex, Hitzewarnsystem, Pollenflug- und Ozonvorhersage] aus gesundheitlicher Sicht - Wie erreichen wir die empfindlichen Bevölkerungsgruppen?)

Kunz-Plapp T, Hackenbruch J, Schipper JW (2016) Factors of subjective heat stress of urban citizens in contexts of everyday life. Nat Hazards Earth Syst Sci 16:977-994

Statistische Ämter der Länder und des Bundes (2019) Internationale Bildungsindikatoren im Ländervergleich, Ausgabe 2020. URL: https://www.statistikportal.de/de/veroeffentlichungen/internationale-bildungsindikatoren-im-laendervergleich-ausgabe-2020 (abgerufen am 27.02.2021)
Robert Koch-Institut (Hrsg.) (2014) Daten und Fakten: Ergebnisse der Studie „Gesundheit in Deutschland aktuell 2012“. Beiträge zur Gesundheitsberichterstattung des Bundes. RKI, Berlin Robert Koch-Institut (Hrsg.) (2019) Erste durch Mücken übertragene West-Nil-Virus-Erkrankung beim Menschen in Deutschland. Gemeinsame Pressemitteilung des Friedrich-LoefflerInstituts, des Bernhard-Nocht-Instituts und des Robert KochInstituts. URL: https://www.rki.de/DE/Content/Service/Presse/Pressemitteilungen/2019/09_2019.html (abgerufen am 27.02.2021)

Rohmert W, Rutenfranz I (1975) Arbeitswissenschaftliche Beurteilung der Belastung und Beanspruchung an unterschiedlichen industriellen Arbeitsplätzen. Bundesministerium für Arbeit und Sozialordnung (Hrsg.), Bonn

Stadt Leipzig, Amt für Statistik und Wahlen (2018) Befragung zum Klimawandel in Leipzig 2018 Ergebnisbericht. URL: https:// static.leipzig.de/fileadmin/mediendatenbank/leipzig-de/ Stadt/02.1_Dez1_Allgemeine_Verwaltung/12_Statistik_ und_Wahlen/Stadtforschung/Ergebnisbericht_Befragung_ zum_Klimawandel_in_Leipzig_2018.pdf (abgerufen am 27.02.2021)

Umweltbundesamt (Hrsg.) (2016) Umweltbewusstsein in Deutschland 2016. Ergebnisse einer repräsentativen Bevölkerungsumfrage. URL: https://www.umweltbundesamt.de/publikationen/umweltbewusstsein-in-deutschland-2016 (abgerufen am 27.02.2021)

Umweltbundesamt (Hrsg.) (2019) Klimawandel und Gesundheit. Tipps für sommerliche Hitze und Hitzewellen https://www. umweltbundesamt.de/publikationen/klimawandel-gesundheit-tipps-fuer-sommerliche-hitze (abgerufen am 27.02.2021)
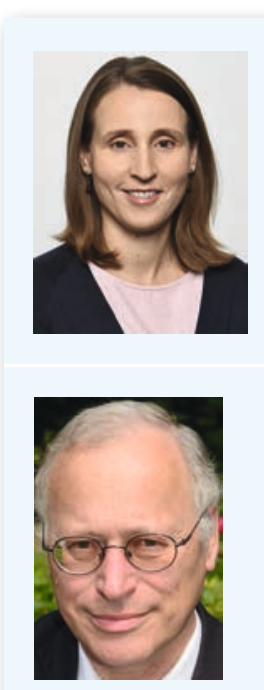

\section{Caroline Schmuker}

Studium der Volkswirtschaftslehre an der Universität Heidelberg. Weiterqualifikation im Fachbereich Epidemiologie an der London School of Hygiene and Tropical Medicine (LSHTM). Berufliche Stationen: 2009 bis 2011 Trainee am Wissenschaftlichen Institut der AOK (WIdO) im Bereich Gesundheitspolitik und Systemanalysen, zwischen 2012 und 2017 wissenschaftliche Mitarbeiterin am IGES Institut Berlin. Seit November 2017 wissenschaftliche Mitarbeiterin im Bereich Qualitäts- und Versorgungsforschung am WIdO.

\section{Prof. Dr. med. Bernt-Peter Robra, MPH}

Epidemiologe und Sozialmediziner, von 1992 bis 2018 Direktor des Instituts für Sozialmedizin und Gesundheitsökonomie der Universität Magdeburg. 

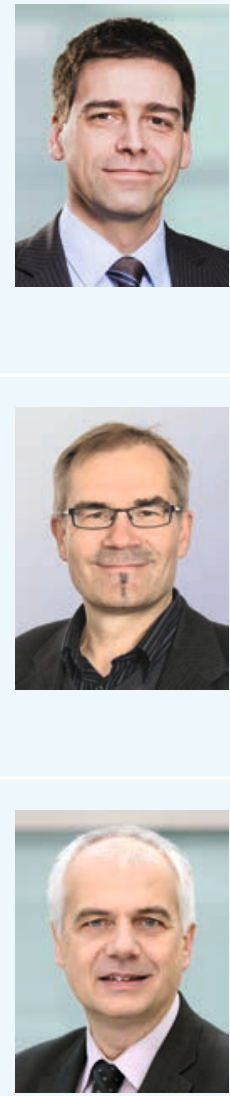

\section{Prof. Dr. med. Kai Kolpatzik, MPH, EMPH}

Kai Kolpatzik ist Arzt und Gesundheitswissenschaftler und arbeitete als Assistenzarzt in der Chirurgie in Krankenhäusern in Freiburg und am Bodensee. Stationen in der Gesundheitswissenschaft waren die Universität Bielefeld - mit Abschluss Master of Public Health und European Master of Public Health - und die Weltgesundheitsorganisation (WHO) in Genf, bevor er 2004 seine Tätigkeit im AOK-Bundesverband aufnahm. Seit 2009 leitet er die Abteilung Prävention im AOK-Bundesverband. Seine Arbeitsschwerpunkte liegen auf der Prävention und Gesundheitsförderung, Gesundheitskompetenz, Ernährungskompetenz, Gesundheitskommunikation, Umwelt und Gesundheit sowie digitalen Gesundheit.

\section{Klaus Zok}

Klaus Zok studierte Geschichte und Sozialwissenschaften an der Ruhr-Universität Bochum mit Abschluss als Diplom-Sozialwissenschaftler. Seit 1992 arbeitet er als wissenschaftlicher Mitarbeiter beim Wissenschaftlichen Institut der AOK (WIdO). Neben der Durchführung von Versichertenund Patientenbefragungen zu verschiedenen Themen der aktuellen gesundheitspolitischen Diskussion (z.B. Reformoptionen und Gestaltungsvorschläge) besteht sein Arbeitsschwerpunkt in der Erstellung von Transparenzstudien zu einzelnen Teilmärkten des Gesundheitssystems (z.B. „IGeL"). Ferner beschäftigt er sich mit strategischen und unternehmensbezogenen Erhebungen und Analysen im GKV-System.

\section{Jürgen Klauber}

Studium der Mathematik, Sozialwissenschaften und Psychologie in Aachen und Bonn. Seit 1990 im Wissenschaftlichen Institut der AOK (WId0) tätig. 1992-1996 Leitung des Projekts GKV-Arzneimittelindex im WId0, 1997-1998 Leitung des Referats Marktanalysen im AOK-Bundesverband. Ab 1998 stellvertretender Institutsleiter und ab 2000 Leiter des WId0. Inhaltliche Tätigkeitsschwerpunkte: Themen des Arzneimittelmarktes und stationäre Versorgung. 